\title{
In-situ measurements of fabrication induced strain in diamond photonic-structures using intrinsic colour centres
}

\author{
Sebastian Knauer $\mathbb{I}^{1,3 凶}$, John P. Hadden (iD $^{1,2,4}$ and John G. Rarity ${ }^{1 凶}$
}

Diamond has established itself as an ideal material for photonics and optomechanics, due to its broad-band transparency and hardness. In addition, colour centres hosted within its lattice such as the nitrogen-vacancy (NV) centre, have become leading candidates for use in quantum information processing, and quantum sensors. The fabrication of nanoscale devices coupled to high quality NVs has been an outstanding challenge due to their sensitivity to magnetic, electric and strain fields within their local environment. In this work, we show how the NV centre's ground state electron spin can be used as an embedded atomic-scale probe of the local strain caused by focused ion beam milling of nanoscale devices. This technique can thus be used to measure, and optimise material and device fabrication processes to allow diamond to reach its full potential.

npj Quantum Information (2020)6:50; https://doi.org/10.1038/s41534-020-0277-1

\section{INTRODUCTION}

The fabrication of photonic structures coupled to single colour centres in diamond has been widely investigated ${ }^{1-5}$, as a key component in many quantum information processing or sensing fields ${ }^{6,7}$. Many attempts to fabricate structures coupled to nitrogen-vacancy (NV) centres have led to unwanted broadening of its spectral lines, reduction in its ground state coherence time, or blinking and ultimately the quenching of the NV centre's fluorescence. The mechanisms causing this are thought to be spectral diffusion or charge state conversion ${ }^{8,9}$ caused by nearby charge traps, as well as homogeneous and inhomogeneous broadening due to spin coupling to paramagnetic defects, and the effect of fabrication induced strain on the electronic and spin states of the NV centre. Here we investigate the effect of such fabrication induced strain on the ground state spin, noting that understanding and quantifying these effects is a necessary step towards the engineering of better quantum devices in diamond.

The effect of strain on the NV centre's optically excited state was previously studied at cryogenic temperatures through photoluminescence $(\mathrm{PL})$ or photoluminescence excitation (PLE) spectroscopy of the centre's zero phonon line $(\mathrm{ZPL})^{10,11}$, as well as at room temperature through pulsed electron spin resonance spectroscopy ${ }^{12}$. Subsequent demonstrations of the sensitivity of the ground state to $\operatorname{strain}^{13}$ encouraged the development of mechanical resonators in diamond, with the aim of demonstrating hybrid opto-mechanical-spin control ${ }^{14,15}$. More recently, the effect of strain on the NV centres infrared singlet transitions has also been studied ${ }^{16,17}$. Although the excited state levels are sensitive to strain, their measurement is restricted to cryogenic temperatures and limited by the excited state lifetime and low PL efficiency of the singlet transitions. Our measurements, focusing on the ground state splitting, allow room temperature measurement of strain.

The NV centre's spin triplet ground state is sensitive to magnetic fields through the Zeeman effect, and to electric fields through the Stark effect ${ }^{18}$. By controlling the interplay between these effects and monitoring the hyperfine structure induced by the NV centre's constituent ${ }^{14} \mathrm{~N}$ spin one nucleus, we are able to enter a regime in which the component of an electric field perpendicular to the NV centre's axis can be measured through its induced splitting of the $m_{\mathrm{s}}=+1, m_{\mathrm{l}}=0$ and $m_{\mathrm{s}}=-1, m_{\mathrm{l}}=0$ (subsequently denoted $|+1,0\rangle,|-1,0\rangle)$ states ${ }^{19,20}$. The Stark effect is caused by the distortion of the electronic orbitals due to an electric field, thus we are able to use the same technique to measure distortion of the NV's orbitals caused by strain within the diamond lattice ${ }^{21}$. The ground state spin susceptibility to strain electric fields perpendicular to the NV centre's symmetry axis is expected to be at least $\sim 50$ times stronger than it is to those parallel to it according to current literature values ${ }^{22}$, which is consistent with our results. Thus, we focus on the sensing of perpendicular strain fields. Because the effect of electric fields and strain fields on the ground state spin are inseparable in our system, we are not currently able to unambiguously determine what contribution to the splitting we observe on NV centres several $\mu \mathrm{m}$ deep is due to electric fields (or band bending) arising from residual Gallium surface layer doping ${ }^{23}$ as opposed to mechanical strain due to Gallium implantation and the creation of vacancies and other defects at the surface ${ }^{11}$. However, noting that the effect of band bending has only been observed for NV centres several tens of $\mathrm{nm}$ below the surface, and because the cleaning process is expected to remove the majority of the implanted layer, we base our analysis on the assumption that the effect is in fact due to strain.

\section{RESULTS}

Theoretical background

The ground state electron spin of an NV centre is described by the Hamiltonian,

$\mathcal{H}_{\mathrm{gs}}=\mathcal{H}_{\text {ogs }}+\mathcal{V}_{\mathrm{Bgs}}+\mathcal{V}_{\mathrm{Egs}}+\mathcal{V}_{\sigma \mathrm{gs}}$

where $\mathcal{H}_{0 \mathrm{gs}}$ is the unperturbed Hamiltonian determining the zero field splitting of the $| \pm 1,0\rangle$ and $|0,0\rangle$ states, and the $\mathcal{V}_{\text {Bgs }}, \mathcal{V}_{\text {Egs }}$

\footnotetext{
'Quantum Engineering Technology Labs, H. H. Wills Physics Laboratory and Department of Electrical and Electronic Engineering, University of Bristol, Bristol BS8 1FD, UK. ${ }^{2}$ Institute for Quantum Science and Technology, University of Calgary, 2500 University Drive NW, Calgary T2N 1N4, Canada. ${ }^{3}$ Present address: Center of Excellence for Quantum Computation and Communication Technology, School of Physics, University of New South Wales, Sydney, NSW 2052, Australia. ${ }^{4}$ Present address: School of Physics and Astronomy, Cardiff University, The Parade, Cardiff CF24 3AA, UK. ${ }^{凶}$ email: knauer.seb@gmail.com; john.rarity@bristol.ac.uk
} 

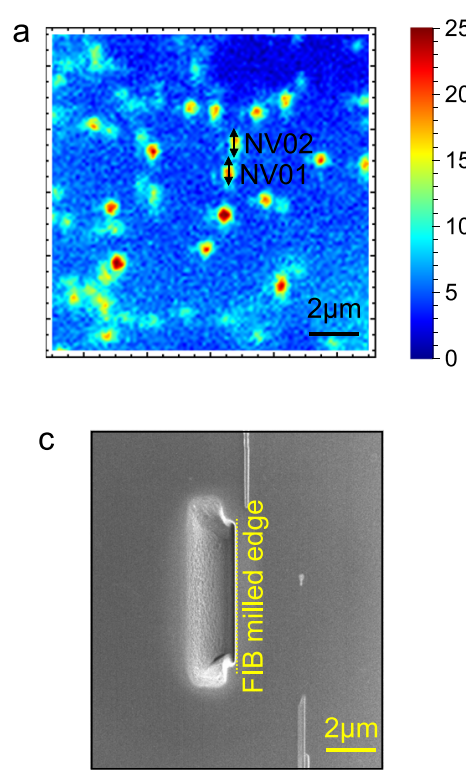
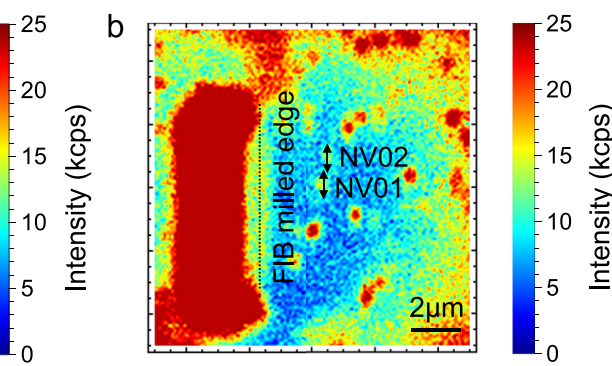

e

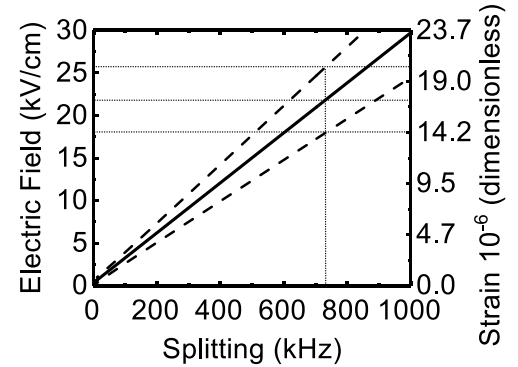

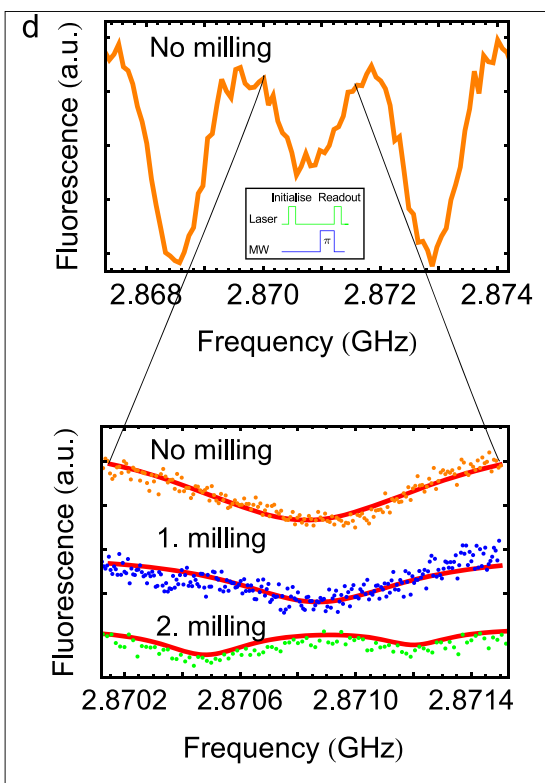

Fig. 1 NV centres near FIB milled interface. $\mathbf{a}$, $\mathbf{b}$ Confocal image in the focal plane of NV01 and NV02 without and with focused ion beam milled edge at a $1.5 \mu \mathrm{m}$ distance. c Corresponding ion-induced secondary electron image. The interface is milled to a depth of $8 \mu \mathrm{m}$ and a width of $6 \mu \mathrm{m}$. d (top) Pulsed optically detected magnetic resonance (ODMR) example for NV02 in the case of no milling (data points are connected for clarity). Inset: Microwave $\pi$-pulse applied between two $532 \mathrm{~nm}$ laser pulses. The photoluminescence in the second laser pulse is detected, and plotted as a function of the microwave frequency. $\mathbf{d}$ (bottom) Pulsed optically detected magnetic resonance (ODMR) of NV02 $| \pm 1,0\rangle$ states. Indicated are no milling, 1 . milling $(1.5 \mu \mathrm{m})$ and 2 . milling $(0.5 \mu \mathrm{m})$ to the reference position of NV01. We cannot resolve any splitting in the unmilled case $0.60 \pm 0.05 \mathrm{MHz}$ (single Lorentzian fit), a broadened Lorentzian fit for 1 . milling to $0.80 \pm 0.05 \mathrm{MHz}$, and a splitting of $720 \pm 4 \mathrm{kHz}$ for 2. milling (double Lorentzian). e The equivalent electric field (left axis) or strain (right axis) associated with a measured splitting. The dashed lines indicate the uncertainty.

and $\mathcal{V}_{\text {ogs }}$ are the perturbations caused by the influence of magnetic (B), electric (E) and strain $(\sigma)$ fields respectively.

Following Dolde et al. ${ }^{19}$, we use second-order perturbation theory to derive linear approximations which allow us to relate fabrication induced splitting of the $|+1,0\rangle,|-1,0\rangle$ states $\Delta \omega_{\text {split, }}$, to the strain equivalent electric field $E_{\perp}$, or strain $\sigma_{\perp}$ perpendicular to the NV centre axis (for the derivation see Supplementary Information 2),

$\Delta \omega_{\text {split }} \approx 2 k_{\mathrm{Egs}}^{\perp} E_{\perp}-\frac{\left(\gamma_{\mu_{\mathrm{B}}} B_{\perp}\right)^{2}}{D_{\mathrm{gs}}}$,

and,

$\Delta \omega_{\text {split }} \approx 2 k_{\sigma g s}^{\perp} \sigma_{\perp}-\frac{\left(\gamma_{\mu_{\mathrm{B}}} B_{\perp}\right)^{2}}{D_{\mathrm{gs}}}$.

Here $D_{\mathrm{gs}}=2.88 \mathrm{GHz}$ is the zero field splitting, $\gamma_{\mu_{\mathrm{B}}}=28 \mathrm{GHzT}^{-1}$ is the gyromagnetic ratio, $B_{\perp}$ is the magnetic field perpendicular to the NV centre axis, $k_{\mathrm{Egs}}^{\perp}=17 \pm 3 \mathrm{~Hz} \mathrm{~cm} \mathrm{~V}^{-1}$ is the electric field susceptibility $^{22}$, and $k_{\text {ogs }}^{\perp}=21.5 \pm 1.2 \mathrm{GHz}$ (per unit strain) is the strain field susceptibility ${ }^{15}$. These approximations are valid where a small magnetic field is carefully aligned perpendicular to the NV centre axis, and the strain is the dominant perturbation.

\section{Sample and measurement method}

In this work we demonstrate the effect of strain field Stark splitting caused by focused ion beam (FIB) milling on the ground state spin of intrinsic 'as grown' single NV centres in bulk electronic grade diamond (4 ppb nitrogen impurity concentration, Element6). We measure strain field-induced shifts when we mill within a few microns of NV centres which initially show no measurable strain field splitting at room-temperature. Two cases of the effect of FIB milling near NV centres are investigated. First we look at the effect of a FIB milled single planar air-diamond interface near NV centres. This simple example allows us to demonstrate the proof of principle use of the NV centre as a sensor for the strain field induced by the fabrication process. Next, we investigate the effect of FIB induced strain fields on pre-characterised single NV centres after the fabrication of solid immersion lenses ${ }^{24}$ (SILs) milled around them. Finally, we use annealing techniques to recover NV centres which have become quenched after FIB milling and in addition show that these NV centres exhibit reduced splitting, indicating reduced strain fields.

\section{Proof of measurement principle}

For the first case, we consider two NV centres, labelled NV01 and NV02, near to a milled air-diamond edge. We show example confocal images of NV01 and NV02 before and after milling (Fig. 1a, b), together with a secondary electron image of the milled edge (Fig. 1c). Prior to the milling we investigated the properties of NV01 and NV02 in the bulk diamond sample. NV01 is located at a depth of $4.3 \mu \mathrm{m}$ and NV02 at $4.5 \mu \mathrm{m}$ from the sample surface. They have a relative distance of about $\Delta x=150 \mathrm{~nm}$ and $\Delta y=$ $1.5 \mu \mathrm{m}$ to each other. Both NV centres' axes fall along the same crystallographic group of orientations in the diamond lattice as determined by polarisation and magnetic field measurements ${ }^{25}$ (Supplementary Information Fig. 3b, 1,2-group), enabling a direct comparison of the measured results.

For each of the NV centres presented here an external magnetic field was precisely aligned perpendicular to the NV centres' axes by minimising the observed Zeeman splitting (Supplementary Information 2.3), using optical magnetic resonance (ODMR) spectra. We estimate a residual magnetic field of $\sim 0.3 \pm 0.1 \mu \mathrm{T}$ in the NV quantisation axes' direction $B_{z}$. Strain fields are then measured by their opposing effects on the $| \pm 1,0\rangle$ hyperfine states, leading to an additional splitting in the ODMR spectrum.

An example pulsed ODMR scan for the unmilled NV02 sample is shown in Fig. 1d (top) with transitions to all three pairs of states, 
while Fig. 1d (bottom, no milling) depicts a scan across the central $| \pm 1,0\rangle$ states. Initially, the $| \pm 1,0\rangle$ hyperfine states of NV01 and NV02 show no splitting with a FWHM of $0.65 \pm 0.04 \mathrm{MHz}$. A sequence of two milling steps was then performed to investigate the effect on the NV centres. The first milling step created a polished air-diamond interface at a distance of $1.5 \mu \mathrm{m}$ to NV01, as shown in Figs. 1b, c. The milling involved an initial coarse cut with a $350 \mathrm{pA}$ beam, followed by a polishing step, using a $150 \mathrm{pA}$ beam. The interface is milled to a depth of $8 \mu \mathrm{m}$ with a width of $6 \mu \mathrm{m}$.

After milling, the sample is cleaned using acid and plasma treatment (Supplementary Information 3) and placed back into the confocal set-up, where the continued fluorescence of NV01 and NV02 is confirmed (Fig. 1b). The milled edge is visible in the confocal scan. Subsequently, a pulsed ODMR scan was performed. The NV02 results are shown in Fig. 1d (bottom) (1. milling). The Lorentzian fit is broadened by $0.15 \pm 0.07 \mathrm{MHz}$ to about $0.80 \pm$ $0.05 \mathrm{MHz}$, but a splitting is not visible.

A second FIB milling followed by acid and plasma cleaning step was carried out to bring the air-diamond interface to a distance of $0.5 \mu \mathrm{m}$ from the position of NV01. After this step we observed that the fluorescence of NV01 quenches and only NV02 is visible. The pulsed ODMR scan (Fig. 1d (bottom), 2. milling) shows a $720 \pm$ $4 \mathrm{kHz}$ splitting of the $| \pm 1,0\rangle$ states (double Lorentzian fit).

Equivalent electric field

To investigate the implications of the measured frequency splittings between the hyperfine states, we estimate the equivalent electric field strength $E_{\perp}$ or mechanical strain field $\sigma_{\perp}$ which would produce such broadening or splittings using Supplementary Eqs. 7 and 10 respectively and as depicted in Fig. 1e. We do not observe a shift of the $| \pm 1,0\rangle$, therefore we conclude that the major sensed strain field is perpendicular to the NV centres' axes. For the case of the 1. milling, where we see a broadening of $0.15 \pm 0.07 \mathrm{MHz}$, we can infer an estimated equivalent electric field (strain field) of $4.7 \pm 2.0 \mathrm{kV} \mathrm{cm}^{-1}(3.7 \pm$ $2.0 \times 10^{-6}$ ), while for the case 2 . milling where we see a splitting of $720 \pm 4 \mathrm{kHz}$, we can infer an estimated equivalent electric field (strain field) of $21.5 \pm 4.0 \mathrm{kV} \mathrm{cm}^{-1}\left(17 \pm 1 \times 10^{-6}\right)$. Note that the uncertainty in these estimates is dominated by the uncertainty in the literature values of the electric and strain field susceptibilities. The equivalent strain field is given in unitless dimensions by convention.

Using a Young's modulus for diamond of $1200 \mathrm{GPa}$, we can see that the stress in the diamond corresponding to these splittings is in the low mega pascals' range (4 MPa $-20 \mathrm{MPa}$ respectively), which is lower than previously reported for measurements on single centres ${ }^{15,26,27}$. The shot noise limited sensitivity, or bandwidth of this measurement is $8.3 \mathrm{kV} \mathrm{cm}^{-1} \mathrm{~Hz}^{-1 / 2}(6.6 \times$ $\left.10^{-6} \mathrm{~Hz}^{-1 / 2}\right)$, however, because the fabrication induced strain is not continuously variable, the minimum detectable equivalent electric field (strain field) of $1.8 \mathrm{kV} \mathrm{cm}^{-1}\left(1.4 \times 10^{-6}\right)$ is potentially more relevant as a measure of the sensitivity of this technique. (Supplementary Information 2.3). Thus, we conclude that the NV centre can be used as a high sensitivity, local measure of lattice deformations ${ }^{28,29}$.

Strain induced by photonic structures

Having demonstrated the ability to sense the FIB induced strain field on NV centres, we apply this technique to investigate the strain field induced by milling solid-immersion lenses (SILs). We observe that the typical fabrication yield of SILs containing optically active NV centres is about $20 \%$. However, although the low yield has important implications for quantum applications of NV centres, there are no thorough studies of this vanishing phenomenon to our knowledge. Our results here suggest the fluorescence quenches might be due to electronic states shifting under mechanical strain fields deep into the diamond lattice.

First, we identify a suitable NV centre, labelled NV03, in the bulk diamond near milled registration markers (Fig. 2). NV03 is located at a depth of $5.6 \mu \mathrm{m}$ and orientated along the crystallographic 1,2group (Supplementary Information Fig. 3b). We characterise NV03 before the SIL milling, using the same methods applied to NV01 and NV02. Figure 2c (black curve) shows the pulsed ODMR spectrum of all NV03 centre's transitions before the SIL milling. A splitting of the central $| \pm 1,0\rangle$ states can't be observed, indicating a low strain field material environment.
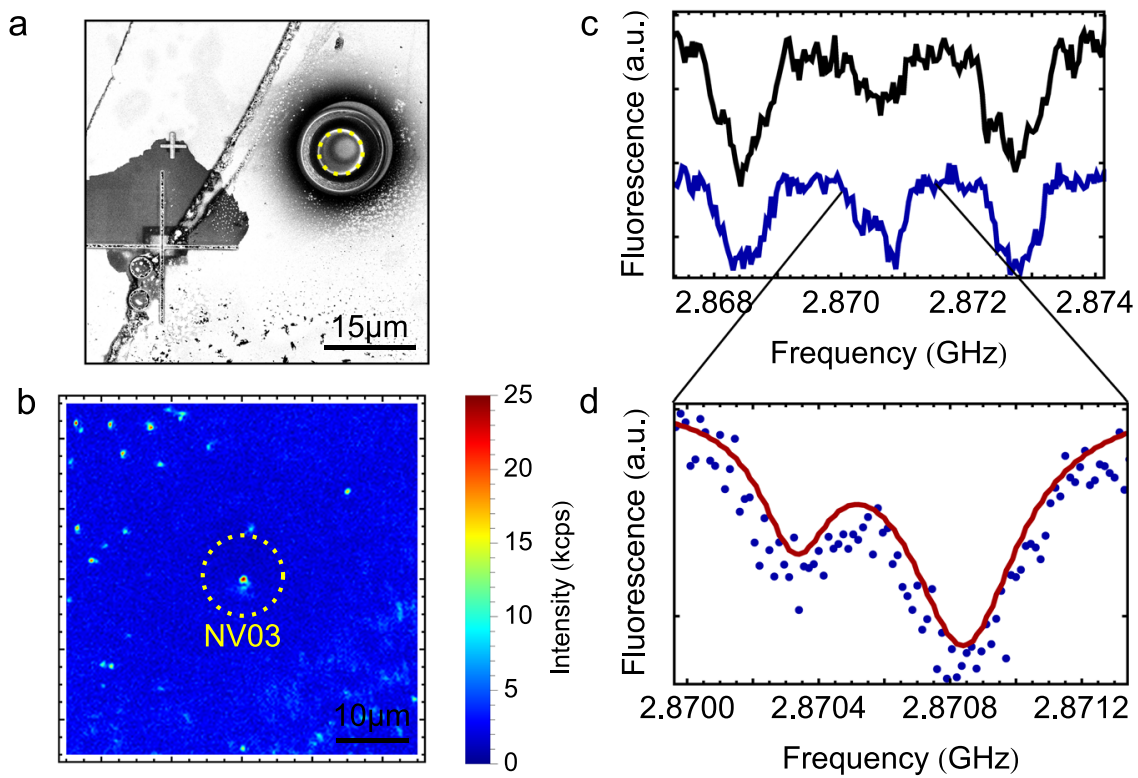

Fig. 2 NV centre underneath a solid immersion lens. a lon-induced secondary electron image of a solid immersion lens near reference markers. b Confocal image of the NV centre (NV03) under solid immersion lens, illustrating placement accuracy less than $100 \mathrm{~nm}$. The lens is marked by the dashed yellow line. NV03 is located in a depth of $5.6 \mu \mathrm{m}$. c Pulsed ODMR trace before (black) and after (blue) milling process of all three transitions (data points are connected for clarity). $\mathbf{d}$ Higher resolution pulsed ODMR scan of the central transitions of the milled sample. We observe no splitting of the central transitions in the unmilled case and a splitting of $510 \mathrm{kHz}$ in the milled case. 
The ion-induced secondary electron image (Fig. 2a) shows the SIL over NV03 after milling. We achieve an alignment accuracy of the centre of the final SIL to NV03 of better than $100 \mathrm{~nm}$, as highlighted in the confocal image Fig. 2b. The extent of the SIL is highlighted with a dashed yellow line. It is designed to achieve a numerical aperture of about 0.9 , so does not constitute a full hemisphere, reducing fabrication damage and fabrication time (SIL fabrication details Supplementary Information 8). Figure 2c also shows a pulsed ODMR scan after the milling (blue). We observe a splitting of the $| \pm 1,0\rangle$ states of $510 \pm 3 \mathrm{kHz}$, calculated from a double Lorentzian fit as illustrated in Fig. $2 \mathrm{~d}$. This splitting relates to an estimated equivalent electrical field strength (strain field) of about $15 \pm 3 \mathrm{kV} \mathrm{cm}^{-1}\left(12.1 \pm 0.7 \times 10^{-6}\right)$, assuming the strain/equivalent electric field is oriented mainly perpendicular to the NV centre's axis. As with the previous NV, the $| \pm 1,0\rangle$ states exhibits no shift, only a splitting.

We studied several other NV centres for which the fluorescence quenched after SIL milling. Figure 3a shows pulsed ODMR scans of four example NV centres (NV04-NV07). Two particular NV centres exhibit splittings before milling, NV05 $770 \pm 5 \mathrm{kHz}$ and NV06 $740 \pm$ $4 \mathrm{kHz}$. These splittings suggest that these single NV centres are in a locally strain field influenced lattice, thus unlikely to survive further milling. This strain/equivalent electric field may originate from perpendicular lattice displacements or from local charges, as these two NV centres are in a particular NV centre rich area. Hence we highlight the strength of this method for the identification of strain free NV centres for quantum information applications.

We investigate the effect of high temperature annealing (see "Methods") on all NV centres studied here and observe that the fluorescence of NV01, NV04, NV06 and NV07 does not return and the fluorescence of NV03 quenches. The surface fluorescence above NV02 increases, limiting further measurements (even after multiple cleaning iterations - see Supplementary Information 3).

However, the fluorescence of NV05 recovers after the annealing process (Fig. 3b). Now we observe a strain splitting of $480 \pm 5 \mathrm{kHz}$ (Fig. 3d), which is nearly half than the observed splitting of the unmilled NV05. The splitting implies an estimated equivalent electrical field strength (strain field) of $14 \pm 3 \mathrm{kV} \mathrm{cm}^{-1}\left(11.4 \pm 6 \times 10^{-6}\right)$.

\section{DISCUSSION}

In this work, we have shown how the NV centre's ground state electron spin can be utilised to measure the strain field induced by damage to the diamond lattice arising from photonic structure milling. In doing so we measure the strain field environment down to to tens of kilohertz of frequency splitting - equivalent to megapascal stress levels with better sensitivity than previously demonstrated by cryogenic excited state spectroscopy methods.

It is not possible to unambiguously ascribe the effects observed to mechanical strain in the diamond lattice rather than electric fields or band bending due to residual doping at the surface. However, such surface chemistry controlled electric charge has till now been shown to have an effect in a range of a few tens of nanometers $8,9,30,31$. The direct measurement of band bending several micrometre deep into non-conductive chemical vapour deposition diamond samples is challenging, due to the nonconductive nature of the material. Future work will attempt to separate unambiguously the two effects.

The techniques presented in this work could also be applied to investigate the strain field (or residual electric field) caused by other fabrication techniques such as reactive ion etching, diamond crystal growth and implantation of NV centres, and as strain induced by bending nanomechanical structures. It should also be noted that NV centres are generated during the implantation and annealing of other colour centres, e.g. the silicon vacancy centre ${ }^{17}$. These techniques may, therefore, provide a tool to understand the local strain and electric field environment of such centres, through measurements of neighbouring NV a

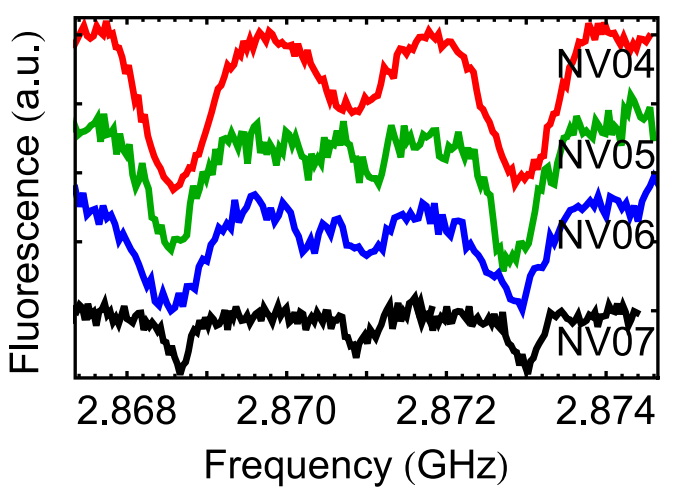

b

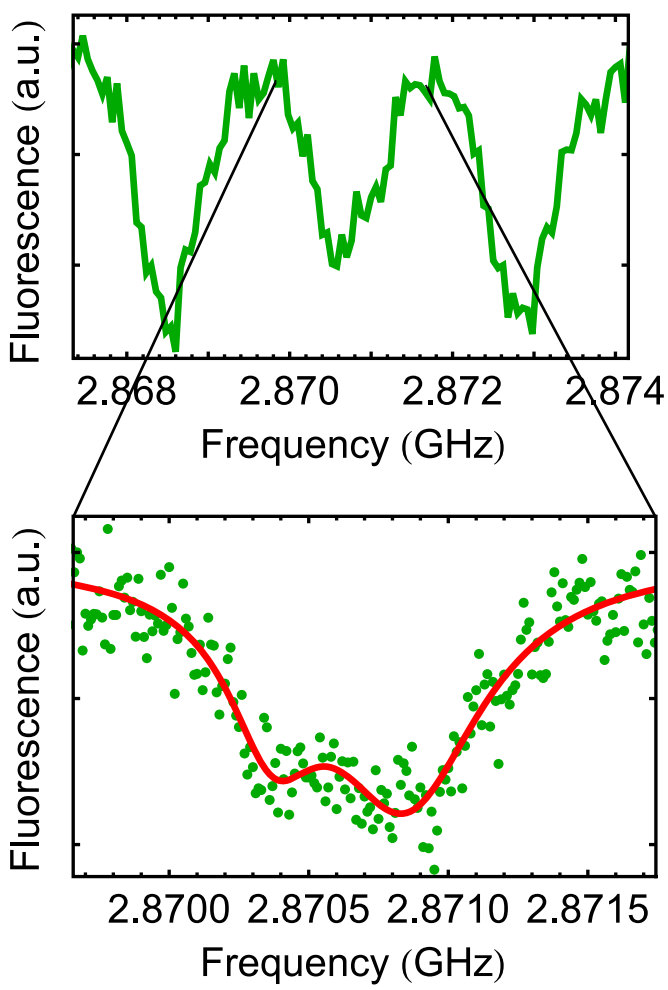

Fig. 3 Pulsed ODMR spectra of various NV centres. a Prior to millings; NV04-NV06 are located in at depth of about $5.5 \mu \mathrm{m}$ and NV07 at $2 \mu \mathrm{m}$. The points are connected for clarity. For NV04 and NV07 we observe no splitting of the $| \pm 1,0\rangle$ states. However, a splitting of $710 \mathrm{kHz}$ for NV05 and $680 \mathrm{kHz}$ for N06 is measured, which indicates local strain or charges. For all candidates NV04-NV07 the fluoresce quenched after the milling of a solid immersion lens, but after annealing NV05 recovered. b Pulsed ODMR spectrum of NV05 after annealing, showing a splitting of the $| \pm 1,0\rangle$. c The zoom shows a higher resolution scan with a double Lorentzian fit, establishing a reduced splitting of $480 \mathrm{kHz}$.

centres. With the use of single NV centres, we are restricted to the measurement of one component of the strain tensor, but by using three or more NV centres in the same region the full strain field tensor could be recovered by the combination of measurements. A comparison of the estimated sensitivity of cryogenic strain measurements suggests that they may be much more sensitive than the methods presented here (see Supplementary 2.5). However, the benefit of our method is that it can be performed without the necessity for cryogenics. Indeed, the recent demonstration of NV centres retaining coherence at temperatures near $700^{\circ} \mathrm{C}$, means it may be possible to perform such measurements during annealing ${ }^{32}$. Further studies investigating the relaxation of 
strain during annealing in more detail may lead to improved post processing recipes for diamond quantum devices. Meanwhile, optimisation of the measurement parameters, and computational methods should enable even higher sensitivity measurements at room temperature ${ }^{4}$.

We show that these measurements are precursors to the vanishing of NV centres due to high strain fields and demonstrate the recovery of NV centres and reduction of strain fields by annealing. We anticipate that these measurements will underpin the development of strain-free photonic structures containing NV centres, suitable for a wide range of nanoscale sensors and quantum information applications.

\section{METHODS}

\section{Optical and electron spin resonance measurements}

$\mathrm{PL}$ and electron spin resonance (ESR) measurements were performed using a home-built scanning confocal setup, equipped with Helmholtz coils for precise magnetic field alignment, and microwave capabilities for NV centre electron spin control (see Supplementary 1 and 2). Pulsed optically detected magnetic resonance (ODMR) measurements, where ESR transitions are identified by the reduction of NV fluorescence intensity consist of an initial spin polarising $532 \mathrm{~nm}$ laser pulse, followed by a single $\pi$ microwave pulse, and a final fluorescence readout laser pulse. The pulse sequence is repeated $\sim 3 \times 10^{6}$ times for each microwave frequency to acquire statistics, and the detected photon detection times are histogrammed leading to about 300 counts in each 25 ns bin during the laser pulse. The total counts in a $300 \mathrm{~ns}$ time window at the start of the laser pulse is normalised against those in another window when it has reached a time averaged steady state. For the evaluation of the length of a $\pi$-pulse, we perform Rabi oscillations, where amplitude of the fluorescent signal is measured as a function of the microwave pulse length $\tau$.

\section{Focused ion beam milling}

For focused ion beam milling and ion-induced secondary imaging a FEIStrata FIB-201 and a FEl-Helios NanoLab 600 with different beam currents are utilised. A more detailed description of the focused ion beam milling is found in the Supplementary 4.

\section{Sample preparation and annealing}

The sample used is an electronic grade [110] top surface bulk diamond (Element6), with a $4 \mathrm{ppb}$ nitrogen impurity concentration. Our measurement identified several candidate NV centres at depths suitable for FIB milling. To decrease the induced strain and to recover quenched NV centres, we perform annealing in a tube furnace (Lenton - LTF 12/50/300). Prior to annealing, we heat the furnace for $12 \mathrm{~h}$ at a temperature of $400^{\circ} \mathrm{C}$ minimise residual particles within the furnace. The sample is cleaned as described in Supplementary 3, then it is placed at the centre of the furnace which is pumped to a pressure lower than $3 \times 10^{-6} \mathrm{mbar}$. The furnace is heated up to $600^{\circ} \mathrm{C}$ and left at this temperature for $30 \mathrm{~min}$, then the temperature is raised to the target temperature of $800^{\circ} \mathrm{C}$ for $7.5 \mathrm{~h}$ and afterwards cooled back down to room temperature. After the annealing the sample is again cleaned, to remove any residual surface graphitisation.

\section{DATA AVAILABILITY}

Data available on request from authors.

Received: 19 November 2019; Accepted: 24 April 2020;

Published online: 02 June 2020

\section{REFERENCES}

1. Chen, Y.-C. et al. Laser writing of coherent colour centres in diamond. Nat. Photonics 11, 77-80 (2016).

2. Hensen, B. et al. Loophole-free Bell inequality violation using electron spins separated by 1.3 kilometres. Nature 526, 682-686 (2015)

3. Nemoto, K. et al. Photonic quantum networks formed from $\mathrm{NV}^{-}$centers. Sci. Rep. 6, 26284 (2016).
4. Santagati, R. et al. Magnetic-field-learning using a single electronic spin in diamond with one-photon-readout at room temperature. Phys. Rev. X 021019, 2160-3308 (2019).

5. Wang, J. et al. Experimental quantum Hamiltonian learning. Nat. Phys. 13, 551-555 (2017)

6. Hausmann, B. J. M. et al. Coupling of NV centers to photonic crystal nanobeams in diamond. Nano Lett. 13, 5791-5796 (2013).

7. Sipahigil, A. et al. An integrated diamond nanophotonics platform for quantumoptical networks. Science 354, 847-850 (2016).

8. Fu, K.-M. C., Santori, C., Barclay, P. E. \& Beausoleil, R. G. Conversion of neutral nitrogen-vacancy centers to negatively charged nitrogen-vacancy centers through selective oxidation. Appl. Phys. Lett. 96, 121907 (2010).

9. Hauf, M. V. et al. Chemical control of the charge state of nitrogen-vacancy centers in diamond. Phys. Rev. B 83, 081304 (2011).

10. Batalov, A. et al. Low temperature studies of the excited-state structure of negatively charged nitrogen-vacancy color centers in diamond. Phys. Rev. Lett. 102, 195506 (2009).

11. Olivero, P. et al. Splitting of photoluminescent emission from nitrogen-vacancy centers in diamond induced by ion-damage-induced stress. N. J. Phys. 15, 043027 (2013).

12. Fuchs, G. D. et al. Excited-state spectroscopy using single spin manipulation in diamond. Phys. Rev. Lett. 101, 117601 (2008).

13. Doherty, M. W. et al. Electronic properties and metrology applications of the diamond NV ${ }^{-}$center under pressure. Phys. Rev. Lett. 112, 047601 (2014).

14. Golter, D. A., Oo, T., Amezcua, M., Stewart, K. A. \& Wang, H. Optomechanical quantum control of a nitrogen-vacancy center in diamond. Phys. Rev. Lett. 116, 143602 (2016).

15. Ovartchaiyapong, P., Lee, K. W., Myers, B. A. \& Jayich, A. C. B. Dynamic strainmediated coupling of a single diamond spin to a mechanical resonator. Nat. Commun. 5, 4429 (2014).

16. Biktagirov, T. B. et al. Strain broadening of the 1042-nm zero-phonon line of the $\mathrm{NV}^{-}$center in diamond: a promising spectroscopic tool for defect tomography. Phys. Rev. B 96, 075205 (2017).

17. Rogers, L. J. et al. Multiple intrinsically identical single-photon emitters in the solid state. Nat. Comm. 5, 4739 (2014).

18. Doherty, M. W. et al. Theory of the ground-state spin of the NV-center in diamond. Phys. Rev. B 85, 205203 (2012).

19. Dolde, F. et al. Electric-field sensing using single diamond spins. Nat. Phys. 7, 459-463 (2011).

20. Iwasaki, T. et al. Direct nanoscale sensing of the internal electric field in operating semiconductor devices using single electron spins. ACS Nano 11, 1238-1245 (2017).

21. Tamarat, T. et al. Stark shift control of single optical centers in diamond. Phys. Rev. Lett. 97, 083002 (2006).

22. Van Oort, E. \& Glasbeek, M. Electric-field-induced modulation of spin echoes of N$\checkmark$ centers in diamond. Chem. Phys. Lett. 168, 529-532 (1990).

23. Broadway, D. A. et al. Spatial mapping of band bending in semiconductor devices using in situ quantum sensors. Nat. Electron. 1, 502-507 (2018).

24. Hadden, J. P. et al. Strongly enhanced photon collection from diamond defect centers under microfabricated integrated solid immersion lenses. Appl. Phys. Lett. 97, 241901 (2010).

25. Doherty, M. W. et al. Measuring the defect structure orientation of a single NVcentre in diamond. N. J. Phys. 16, 063067 (2014).

26. Grazioso, F., Patton, B. R., Delaney, P., Markham, M. L., Twitchen, D. J. \& Smith, J. M. Measurement of the full stress tensor in a crystal using photoluminescence from point defects: the example of nitrogen vacancy centers in diamond. Appl. Phys. Lett. 103, 101905 (2013).

27. Neumann, P. et al. Excited-state spectroscopy of single NV defects in diamond using optically detected magnetic resonance. N. J. Phys. 11, 013017 (2009).

28. Deák, P., Aradi, B., Kaviani, M., Frauenheim, T. \& Gali, A. Formation of NV centers in diamond: a theoretical study based on calculated transitions and migration of nitrogen and vacancy related defects. Phys. Rev. B 89, 075203 (2014).

29. Pinto, H., Jones, R., Palmer, D. W., Goss, J. P., Briddon, P. R. \& Öberg, S. On the diffusion of NV defects in diamond. Phys. Status Solidi Appl. Mater. Sci. 209, 1765-1768 (2012).

30. Denisenko, A., Romanyuk, A., Pietzka, C., Scharpf, J. \& Kohn, E. Surface damages in diamond by $\mathrm{Ar} / \mathrm{O} 2$ plasma and their effect on the electrical and electrochemical characteristics of boron-doped layers. J. Appl. Phys. 108, 074901 (2010).

31. Schreyvogel, C., Polyakov, V., Wunderlich, R., Meijer, J. \& Nebel, C. E. Active charge state control of single NV centres in diamond by in-plane Al-Schottky junctions. Sci. Rep. 5, 12160 (2015).

32. Liu, G.-Q., Feng, X, Wang, N., Li, Q. \& Liu, R.-B. Coherent quantum control of nitrogen-vacancy center spins near 1000 kelvin. Nat. Commun. 10, 1344, (2019). 


\section{ACKNOWLEDGEMENTS}

This work has been funded by the project "WASPS" (Wavelength-tunable Advanced Single Photon Sources), which acknowledges the financial support within the Seventh Framework Programme for Research of the European Commission, under FET-Open grant number: FP7-618078. J.G.R. is sponsored under EPSRC grant number EP/M024458/1. S.K. acknowledges the support of Tom Scott and Peter Heard from the IAC Bristol; and of Antony Carrington and Carsten Putzke from the Correlated Electron Systems group Bristol.

\section{AUTHOR CONTRIBUTIONS}

S.K. conceived and designed the experiments in consultation with J.G.R. J.P.H. performed the programming of the software for the confocal and spin measurements and the spin simulation. S.K. built the setup, performed experiments and analysed the data. The paper was written with feedback from all authors.

\section{COMPETING INTERESTS}

The authors declare no competing interests.

\section{ADDITIONAL INFORMATION}

Supplementary information is available for this paper at https://doi.org/10.1038/ s41534-020-0277-1.
Correspondence and requests for materials should be addressed to S.K. or J.G.R.

Reprints and permission information is available at http://www.nature.com/ reprints

Publisher's note Springer Nature remains neutral with regard to jurisdictional claims in published maps and institutional affiliations.
(C) (i) Open Access This article is licensed under a Creative Commons Attribution 4.0 International License, which permits use, sharing, adaptation, distribution and reproduction in any medium or format, as long as you give appropriate credit to the original author(s) and the source, provide a link to the Creative Commons license, and indicate if changes were made. The images or other third party material in this article are included in the article's Creative Commons license, unless indicated otherwise in a credit line to the material. If material is not included in the article's Creative Commons license and your intended use is not permitted by statutory regulation or exceeds the permitted use, you will need to obtain permission directly from the copyright holder. To view a copy of this license, visit http://creativecommons. org/licenses/by/4.0/.

(c) The Author(s) 2020 